\title{
THYSANOPTERA (PHLAEOTHRIPIDAE) EN HUERTOS DE AGUACATE EN NAYARIT, MÉXICO
}

\section{Octavio Jhonathan CAMBERO-CAMPOS, ${ }^{1,2}$ GERARdo A. SOTO-RODRÍGUEZ ${ }^{3,4}$ y AXEL P. RETANA-SALAZAR ${ }^{3,5}$}

\author{
${ }^{1}$ Unidad Académica de Agricultura, ${ }^{2}$ Posgrado en Ciencias Biológico Agropecuarias; Universidad Autónoma de \\ Nayarit, Xalisco, Nayarit, México, carretera Tepic-Compostela Km 9. C.P. 63155 < jhony695@gmail.com> \\ ${ }^{3}$ Centro de Investigación en Estructuras Microscópicas (CIEMIC), Ciudad de la Investigación, Universidad de \\ Costa Rica 2060. \\ ${ }^{4}$ Hypericum Pharma, Pavas, San José, Costa Rica. \\ ${ }^{5}$ Escuela de Nutrición, Facultad de Medicina, Ciudad de la Investigación, Universidad de Costa Rica 2060.
}

Recibido: 12/12/2013; aceptado: 06/05/2015

\begin{abstract}
Cambero-Campos, O. J., Soto-Rodríguez, G. A. \& Retana-Salazar, A. P. 2015. Thysanoptera (Phlaeothripidae) en huertos de aguacate en Nayarit, México. Acta Zoológica Mexicana (n. s.), 31(2): 178-182.
\end{abstract}

RESUMEN. Se realizó un trabajo para identificar y cuantificar las especies de Phlaeothripidae asociadas a cultivares de aguacate en Nayarit, México. Los resultados permitieron identificar un total de 10 especies, dos en Idolothripinae y ocho en Phlaeothripinae. La especie más representativa fue Haplothrips gowdeyi. Asimismo, se presenta información sobre las localidades y datos de recolecta y acerca de los géneros y cada una de las especies encontradas.

Palabras clave: Thysanoptera, Phlaeothripidae, aguacate, México.

\section{INTRODUCCIÓN}

La familia Phlaeothripidae se compone de alrededor de 3520 especies en 460 géneros (Mound et al. 2013) y se divide a su vez en dos subfamilias Idolothripinae y Phlaeothripinae (Mound \& Marullo 1996). Se han realizado diversos trabajos de monitoreo e identificación de las especies fitófagas de Thysanoptera asociadas a huertos de aguacate en Nayarit, México (Cambero-Campos et al. 2010 y Cambero-Campos et al. 2011a) de géneros depredadores como Aeolothrips Haliday, 1836, Erythrothrips Moulton, 1911, Franklinothrips Back, 1912, Scolothrips Hinds, 1902, Leptothrips Hood, 1909 y Trybomia Karny, 1911 (Cambero-Campos et al. 2011c) y la descripción de nuevas especies (Cambero-Campos et al. 2011b). Estos trabajos incrementaron el conocimiento de Thysanoptera asociados a este cultivo y particularmente en este estado de México, por ser el segundo lugar a nivel nacional en cuanto a volumen de producción anual y superficie plantada, con cerca de 2703 ha (Cambero-Campos et al. 2011c). El objetivo de este trabajo fue identificar otras especies de Tubulifera asociadas al cultivo del aguacate en Nayarit.
Cambero-Campos, O. J., Soto-Rodríguez, G. A. \& Retana-Salazar, A. P. 2015. Thysanoptera (Phlaeothripidae) in avocado orchards in Nayarit, Mexico. Acta Zoológica Mexicana (n. s.), 31(2): 178-182.

ABSTRACT. A work was realized to identify and quantify the associated Phlaeothripidae species to cultivars of avocado in Nayarit, Mexico. The results allowed identified a total of 10 species, two in Idolothripinae and eight in Phlaeothripinae. Haplothrips gowdeyi was the most representative. Information about locations was provided, including data and taxonomic or biological details of each species.

Key Words: Thysanoptera, Phlaeothripidae, avocado, Mexico.

\section{MATERIALES Y MÉTODOS}

Se realizaron muestreos semanales por medio de redeo y derribo, de enero a diciembre del 2008. Los muestreos se realizaron en cinco huertos comerciales de aguacate Persea americana Mill. (Lauraceae) ubicadas en los municipios de Tepic, Xalisco y San Blas, Nayarit, México En Tepic, las recolectas se hicieron en dos huertos con el cultivar "Hass", la primera en Ejido Camichin de Jauja llamada “Tintilagua' $\left(21^{\circ} 29 \times 18.5^{\prime}\right.$ ”N, 10446 × 18.4”W, 1117 msnm) y la segunda en 'El Fortín' en Ejido Venustiano Carranza $\left(21^{\circ} 31 \times 00.5^{\prime}\right.$ N, $104^{\circ} 58 \times 57.8^{\prime}$ ”W, 1099 msnm). En Xalisco, se muestrearon dos huertos con el cultivar "Hass", 'La Chapula' Ejido Xalisco $\left(21^{\circ} 25 \times\right.$ 09.4” N, 104 54 × 53.9”'W, 1035 msnm) y 'La Carbonera' Ejido El Cuarenteño $\left(21^{\circ} 27 \times 34.1^{\prime}\right.$ ” N, $105^{\circ} 00 \times 19.1^{\prime}$ ”W, $1787 \mathrm{msnm})$. Finalmente en San Blas, las muestras se tomaron del huerto 'El Cedro' Ejido Mecatan (21 $32 \times$ 47.4” N, $105^{\circ} 08 \times 23.1^{\prime \prime} \mathrm{W}, 384 \mathrm{msnm}$ ), con los cultivares 'Choquette' y 'Hall'. Los thrips recolectados se colocaron en viales de 5 cc conteniendo alcohol al 70\% y se trasladaron al Laboratorio de Entomología de la Universidad Autónoma Agraria Antonio Narro, donde se montaron en 
láminas en Bálsamo de Canadá (Mound \& Marullo 1996) y se identificaron de acuerdo a las claves de Mound \& Marullo (1996) y Soto-Rodríguez \& Retana-Salazar (2003).

\section{RESULTADOS}

En los muestreos realizados se encontraron diez especies de Tubulifera, asociadas a los huertos de aguacate; dos especies para la subfamilia Idolothripinae y ocho especies para la subfamilia Phlaeothripinae. La especie más abundante fue Haplothrips gowdeyi (13 individuos) la cual se asocia a pastos. En contraste Adraneothrips lepidus, Hoplandrothrips jennei y Eurythrips sp. se recolectaron 4 individuos (Cuadro 1). El género con más especies fue Adraneothrips (3) seguido de Karnyothrips con dos.

\section{PHLAEOTHRIPIDAE}

\section{SUBFAMILIA IDOLOTHRIPINAE}

Allothrips Hood, 1908

Allothrips megacephalus mexicanus Stannard, 1955

Material examinado. Hembra macróptera recolectada en San Blas, huerta 'El Cedro' en Ejido Mecatan (28. v.2008).

Comentarios. El género comprende un total de 23 especies de varias partes del mundo (Mound 2007). Se alimentan de esporas de diferentes especies de hongos (Mound 1972). Este mismo autor, indica que Stannard describió esta forma a partir de siete hembras recolectadas en Acapulco, de los cuales dos especímenes presentaban estados intermedios entre mexicanus y megacephalus. El único espécimen recolectado en este estudio se incluyó en la subespecie mexicanus, pero posee algunos caracteres intermedios entre ésta y $A$. brasilianus.

Cuadro 1. Especies y cantidad de individuos recolectados en cinco huertos de aguacate de los municipios de Xalisco, Tepic y San Blas, Nayarit.

\begin{tabular}{|c|c|c|}
\hline Especie & $\hat{\partial}$ & 우 \\
\hline $\begin{array}{l}\text { Allothrips megacephalus mexicanus Stannard, } \\
1955\end{array}$ & 0 & 1 \\
\hline Gastrothrips Hood, 1912 & 0 & 1 \\
\hline $\begin{array}{l}\text { Adraneothrips biadenes Mound \& Marullo, } \\
1996\end{array}$ & 0 & 3 \\
\hline Adraneothrips fuscicollis Hood, 1925 & 0 & 2 \\
\hline Adraneothrips lepidus Hood, 1950 & 0 & 4 \\
\hline Haplothrips gowdeyi Franklin, 1908 & 0 & 13 \\
\hline Hoplandrothrips jennei Jones, 1912 & 4 & 0 \\
\hline Karnyothrips brimleyi Hood, 1938 & 0 & 1 \\
\hline Karnyothrips venustus Moulton, 1941 & 0 & 2 \\
\hline Eurythrips Hinds, 1902 & 0 & 4 \\
\hline Totales & 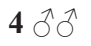 & 31 우우 \\
\hline
\end{tabular}

Gastrothrips Hood, 1912

\section{Gastrothrips sp.}

Material examinado. Hembra macróptera recolectada en San Blas, huerta 'El Cedro' en Ejido Mecatan (05. iii.2008).

Comentarios. Mound (1974) presenta una clave para 16 especies de Gastrothrips y comenta que uno de los mayores problemas en la identificación de especies de este género es que se cuenta con pocos especímenes (macróptera, micróptera ó áptera) lo que se dificulta la comparación entre especies. Mound \& Palmer (1983) presentan un listado de 32 especies e informan para México a G. corvus Priesner y G. pueblae Johansen.

\section{SUBFAMILIA PHLAEOTHRIPINAE}

\section{Adraneothrips Hood, 1925}

El género incluye un total de 77 especies (Dang et al. 2013). Se alimentan de hongos y es posible encontrarlos en hojarasca y en hojas colgantes muertas (Mound \& Marullo 1996, Soto-Rodríguez \& Retana-Salazar, datos sin publicar). Las especies del género comparten un carácter que es poco usual dentro de Phlaeothripinae, que el par de setas accesorias en el tergo IX, entre la seta B1 y B2 son largas, tanto como la seta B1 (Dang et al. 2013, Mound et al. 2013). Es un género cosmopolita, ya que se informan seis especies del Viejo Mundo (dos de África, tres de India y una de Australia), además de las que se conocen para el Nuevo Mundo y especialmente en el Neotrópico (Dang et al. 2013). Posiblemente se presenten tres grupos divergentes: grupo alternatus, grupo bellus y grupo uniformis (Mound \& Marullo 1996).

\section{Adraneothrips biadenes Mound \& Marullo, 1996}

Material examinado. 3 hembras macrópteras. 1 recolectada en San Blas, huerta 'El Cedro' en Ejido Mecatan (27.viii.2008) y 2 $9+$ recolectadas en la huerta El Fortin' en Ejido Venustiano Carranza (08 y 29.x.2008).

Comentarios. Originalmente descrito a partir de material recolectado en Monteverde y en la Península de Osa en Costa Rica, Mound \& Marullo (1996) indican que las hembras de esta especie son cercanas a A. fuscicollis. Sin, embargo las setas anteriores del pronoto son muy cortas. Por su parte, Álvarez et al. (2007) presentan una clave para las especies de Adraneothrips de Centroamérica y separan a $A$. biadenes de $A$. fuscicollis por la longitud de las setas anteroangulares del pronoto.

Adraneothrips fuscicollis Hood, 1925

Material examinado. 2 hembras macrópteras. $2 q q$ recolectadas en San Blas, huerta ‘El Cedro’ en Ejido Meca$\tan$ (13.viii.2008 y 22.x.2008).

Comentarios. esta especie ha sido colectada en pastizales, hojarasca y frutos secos. Es muy cercana a A. alternatus, pero se diferencia de ésta por la presencia de sensilas campaniformes en la pelta, los mismos que $A$. fuscicollis (Mound \& Marullo 1996, Álvarez et al. 2007). 
Adraneothrips lepidus Hood, 1950

Material examinado. 4 hembras macrópteras recolectadas en San Blas, huerta 'El Cedro' en Ejido Mecatan (05. viii.2008, 08.x.2008 y 03-08.xii.2008).

Comentarios. Esta especie fue descrita para Brasil (Mound \& Marullo 1996).

\section{Haplothrips Amyot \& Serville, 1843}

Haplothrips gowdeyi Franklin, 1908

Material examinado. 13 hembras macrópteras. 4 우 recolectadas en San Blas, huerta 'El Cedro' en Ejido Mecatan (05.iii.2008 y 05.viii.2008); 5 우 0 recolectadas en Xalisco, tres en la huerta La Carbonera' en Ejido El Cuarenteño (09.vii.2008 y 06.viii.2008) y dos en la huerta 'La Chapula' en Ejido Xalisco (20.ii.2008 y 19.x.2008); 4 우 recolectadas en Tepic, tres en la huerta en Ejido Camichin de Jauja llamada 'Tintilagua' (06.ii.2008 y 19.iii.2008) y otra en la huerta El Fortin' en Ejido Venustiano Carranza (23.i.2008).

Comentarios. Haplothrips es el tercer género más grande dentro de Thysanoptera (Mound \& Zapater 2003) con 250 especies distribuidas en el mundo y menos de 20 se consideran endémicas del Nuevo Mundo (Mound \& Minaei 2007). Esta especie fue la más abundante, lo cual coincide con otros trabajos (Soto-Rodríguez et al. 2009). Fue descrita originalmente de Barbados, pero es posible que sea originaria de África (Mound \& Marullo 1996), está asociada a diferentes especies de flores y presenta una amplia distribución (Mound \& Minaei 2007).

\section{Hoplandrothrips Hood, 1912}

Es un género muy diverso por su estructura corporal (Mound \& Marullo, 1996). Existen 106 especies, de las cuales 48 han sido descritas para el Nuevo Mundo: 22 para Norteamérica, nueve para México y 17 de la región Neotropical (Mound \& Tree 2013).

\section{Hoplandrothrips jennei Jones, 1912}

Material examinado. 4 machos macrópteros. 3 $\widehat{ึ}$ recolectados en Tepic, en Ejido Camichin de Jauja en la huerta llamada 'Tintilagua' (26.iii.2008, 14.v.2008 y 03.ix.2008) y $1 \overbrace{}^{\lambda}$ recolectado en Xalisco, en la huerta 'La Chapula' en Ejido Xalisco (09.i.2008).

Comentarios. el material recolectado corresponde a machos grandes, con setas fuertes y gruesas en la parte del propleuron, pronoto ancho con forma de armadura y presentan el par de setas anteroangulares del pronoto muy desarrolladas (Mound \& Tree 2013, Mound et al. 2013).

\section{Karnyothrips Watson, 1923}

Karnyothrips es un género principalmente del Nuevo Mundo (Mound \& Marullo 1996), varios taxones registran este nombre, ya que las especies de este género no son homogéneas en sus caracteres fundamentales (Retana-Salazar \& Soto-Rodríguez 2007). Por lo que se confunde con el género Apterygothrips (Retana-Salazar \&
Soto-Rodríguez 2007, Cambero-Campos et al. 2011b). Johansen \& Mojica (1993) reportan seis especies para México, incluyendo la descripción de K. tepoztlanensis y claves para las mismas. En contraste, Cambero-Campos et al. (2011b) describen una nueva especie, K. maurilia, en huertos de aguacate en Nayarit y la especie más cercana a ésta es K. brimleyi Hood. En base a la configuración de la antena en cuanto a la presencia de dos conos sensoriales en el antenómero III.

\section{Karnyothrips brimleyi Hood, 1938}

Material examinado. 1 hembra macróptera recolectada en Xalisco, en la huerta La Carbonera' en Ejido El Cuarenteño (27.ii.2008).

Comentarios. esta especie fue descrita del norte de Carolina y Florida, Estados Unidos (Mound \& Marullo 1996). Karnyothrips venustus Moulton, 1941

Material examinado. 2 hembras macrópteras recolectadas en Xalisco, en la huerta La Carbonera' en Ejido El Cuarenteño (06.i.2008 y 23.iv.2008).

Comentarios. esta especie se conoce únicamente de Brasil (Mound \& Marullo 1996).

\section{Eurythrips Hinds, 1902}

\section{Eurythrips sp.}

Material examinado. 4 hembras macrópteras. 3 + $q$ recolectadas en San Blas, huerta 'El Cedro' en Ejido Meca$\tan (20.1 i .2008,19$. iii.2008 y 28.v.2008) y 1 q recolectada en Tepic, en la huerta El Fortin' en Ejido Venustiano Carranza (27.ii.2008).

Comentarios. De acuerdo con Mound (1976), este género es difícil de distinguir de otros tales como Erkosotrips, Terthrothrips, Orthothrips, Copiothrips, Phragmothrips y Pleurothrips. Ninguna de las especies de Eurythrips posee reticulación en la cabeza cerca de las setas posoculares. Sin embargo, pocas especies presentan una débil reticulación en la parte media de la cabeza. Para México se informan a E. batesi Watson y E. dissimilis Hood (Mound 1976).

\section{DISCUSIÓN}

El muestreo utilizando métodos de recolecta generales con repeticiones semanales indica que se trata de especies poco frecuentes a raras (Álvarez et al. 2007). En los sistemas tropicales es común encontrar una amplia diversidad, donde la misma no registra una dominancia clara de alguna especie y la mayor parte se ubican en el ámbito de raras o poco frecuentes (Guttman 1999). La rareza de las especies es un fenómeno común que afecta el trabajo taxonómico y ecológico, en el primer caso es usual encontrar pocos especímenes de especies nuevas que muchas veces tardan décadas en ser descritas esperando hallar nuevos especímenes. En los estudios ecológicos los números ba- 
jos afectan el uso de pruebas estadísticas robustas y muchas veces estas especies deben incluirse tan solo como observaciones. La rareza de las especies se ve afectada por múltiples factores naturales y es un fenómeno ecológico común y poco estudiado (Gaston 1994). En los estudios de agroecosistemas es frecuente que se desestimen las especies raras, puesto que no son de utilidad en los análisis de daño o asociación de especies. No obstante, la presencia de especies raras en estos sistemas es un factor de importancia en la comprensión de factores ecológicos micro. En la actualidad los estudios ecológicos se centran en la necesidad de establecer los ámbitos de distribución de las especies y de aproximar mediante programas informáticos especializados la distribución probable de las especies (Retana-Salazar et al. 2012). Actualmente, informar nuevas localidades para las especies se convierte en un dato de mucha utilidad en la proyección de colonización de especies invasoras la cual se ha incrementado con el cambio climático afectando sobre todo a la fauna de las zonas tropicales (Deutsch et al. 2008). En México se han encontrado recientemente especies que solo se hallaban en América del Sur como es el caso de Ethirothrips firmus conocido solo de la serie tipo de Sao Paulo, Brasil de 1952, el cual es el único espécimen recolectado en México en Nayarit (Valenzuela-García et al. 2011). Los modestos informes de nuevas distribuciones de las especies como de su presencia en agroecosistemas son de utilidad en la aplicación de modernas tecnologías de estudio de las especies y su capacidad de dispersión, de igual forma el estudio de este material contribuye en el estudio de las variaciones morfológicas y la constancia de los caracteres de las especies (Valenzuela-García et al. 2011).

En la actualidad numerosas investigaciones se enfocan en los problemas que tendrán los agroecosistemas con el cambio climático y las variaciones atmosféricas que propicien desplazamientos en las poblaciones de insectos, en la mayoría de los casos los problemas se incrementan dándole ventajas reproductivas a algunas especies de climas cálidos (Deka et al. 2009). Estos datos resaltan la importancia de mantener un conocimiento actualizado acerca de las especies asociadas a los agroecosistemas de las zonas tropicales.

Las especies de Karnyothrips suelen ser depredadoras lo cual explica parcialmente que sean menos abundantes, pues sus poblaciones dependen del recurso disponible (Ricklefs 1984). La mayor parte de las especies registradas pertenecen a grupos en los cuales se explotan los pastos y las ramas en descomposición como hábitat.

Por otra parte, algunas de estas especies pertenecen a grupos que han sido asociados a sitios no perturbados de vegetación nativa (Retana-Salazar 2006). Por ejemplo, Allothrips megacephalus, Adraneothrips y Eurythrips. En relación a Adraneothrips que presenta gran diversidad de especies y hábitats, $A$. gandoca se presenta en hábitats poco perturbados (Álvarez et al. 2007). Esta especie ha sido también encontrada en cafetales de Costa Rica en los que el tratamiento del cultivo es orgánico, con lo que parece ser que la especie se asocia a sistemas poco alterados.

AGRADECIMIENTOS. A la Universidad Autónoma Agraria Antonio Narro y Universidad Autónoma de Nayarit por las facilidades y apoyo brindado para la realización de la presenta investigación.

\section{LITERATURA CITADA}

Álvarez, C., Sánchez, C. \& Retana-Salazar, A. P. 2007. Una nueva especie de Adraneothrips (Thysanoptera, Tubulifera, Phlaeothripidae). Iheringia Série Zoologia, 97: 243-245.

Cambero-Campos, O. J., Johansen, R., Retana-Salazar, A. P., García, O., Cantú, M. \& Carvajal, C. 2010. Thrips (Thysanoptera) del aguacate (Persea americana) en Nayarit, México. Revista Colombiana de Entomología, 36: 47-51.

Cambero-Campos, O. J., Johansen-Naime, R., García-Martínez, O., Cerna-Chávez, E., Robles-Bermúdez, A. \& Retana-Salazar, A. P. 2011a. Species of thrips (Thysanoptera) in avocado orchards in Nayarit, México. Florida Entomologist, 94: 982-986.

Cambero-Campos, O. J., García-Martínez, O., Johansen, R. \& Retana-Salazar, A. P. 2011b. Una nueva especie del género Karnyothrips (Phlaeothripidae: Tubulifera). Acta Zoológica Lilloana, 55: 11-15.

Cambero-Campos, O. J., Johansen-Naime, R., García-Martínez, O., Cantú-Sifuentes, M., Cerna-Chávez E. \& Retana-Salazar, A. P. 2011c. Especies depredadoras de trips (Thysanoptera) asociadas a huertas de aguacate en Nayarit, México. Acta Zoológica Mexicana (n. s.), 27: 115-121.

Dang, Li-H., Mound, L. A. \& Qiao, Ge-Xia. 2013. Leaf-litter thrips of the genus Adraneothrips from Asia and Australia (Thysanoptera, Phlaeothripinae). Zootaxa, 3716: 001-021.

Deka, S., Byjesh, K., Kumar, U. \& Choudhary, R. 2009. Climate change and impacts on crop pests-A critique. ISPRS Archives XXXVIII-8/W3 Workshop Proceedings: Impact of Climate Change on Agriculture, 17-18 December, Ahmedabad, India pp: 147-149.

Deutsch, C. A., Tewksbury, J. J., Huey, R. B., Sheldon, K. S., Ghalambor, C. K., Haak, D. C. \& Martin, P. R. 2008. Impacts of climate warming on terrestrial ectotherms across latitude. PNAS, 105: 6668-6672.

Gaston, K. J. 1994. Rarity. Chapman \& Hall, London, UK, 205pp.

Guttman, B. S. 1999. Biology. The Structure of Biological Communities. WCB McGraw-Hill. Pp. 556-578.

Johansen, R. M. \& Mojica-Guzmán, A. M. 1993. Nuevos thrips tubulíferos (Insecta: Thysanoptera) de México. XV. Anales del Instituto de Biología Universidad Nacional Autónoma de México, Serie Zoología, 64: 17-37.

Mound, L. A. 1972. Polytypic species of spore-feeding Thysanoptera in the genus Allothrips Hood (Phlaeothripidae). Journal of Australian Entomological Society, 11: 23-36.

Mound, L. A. 1974. The Nesothrips complex of spore-feeding Thysanoptera (Phlaeothripidae: Idolothripinae). Bulletin of the British Museum (Natural History). Entomology, 31: 109-188.

Mound. L. A. 1976. American leaf-litter Thysanoptera of the genera Erkosothrips, Eurythrips y Terthrothrips (Phlaeothripidae: 
Phlaeothripinae). Bulletin of the British Museum (Natural History). Entomology, 35: 25-64.

Mound, L. A. 2007. New Australian spore-feeding Thysanoptera (Phlaeothripidae: Idolothripinae). Zootaxa, 1604: 53-68.

Mound, L. A. \& Palmer, J. M. 1983. The generic and tribal classification of spore-feeding Thysanoptera (Phlaeothripidae: Idolothripinae). Bulletin of the British Museum (Natural History). Entomology, 46: 1-174.

Mound, L. A. \& Marullo, R. 1996. The thrips of Central and South America: An Introduction (Insecta: Thysanoptera). Memoirs on Entomology. 487 pp.

Mound, L. A. \& Zapater, M. C. 2003. South American Haplothrips species (Thysanoptera: Phlaeothripidae), with a new species of biological control interest to Australia against weedy Heliotropium amplexicaule (Boraginaceae). Neotropical Entomology, 32: 437-442.

Mound, L. A. \& Minaei, K. 2007. Australian thrips of the Haplothrips lineage (Insecta: Thysanoptera). Journal of Natural History, 41: 2919-2978.

Mound, L. A., Dang, Li-H. \& J. Tree, D. 2013. Genera of fungivorous Phlaeothripinae (Thysanoptera) from dead branches and leaf-litter in Australia. Zootaxa, 3681: 201-224.

Mound, L. A. \& J. Tree, D. 2013. Fungus-feeding thrips from Australia in the worldwide genus Hoplandrothrips (Thysanoptera: Phlaeothripinae). Zootaxa, 3700: 476-494.
Retana-Salazar, A. P. 2006. Estudio Preliminar de Thrips como Bioindicadores (Insecta: Tubulifera). Métodos en Ecología y Sistemática, 1: 10-13.

Retana-Salazar, A. P. \& Soto-Rodríguez, G. A. 2007. Revisión taxonómica del grupo Haplothrips-Karnyothrips (Thysanoptera: Phlaeothripidae). Revista de Biología Tropical, 55: 627-635.

Retana-Salazar, A. P., Garita-Cambronero, J., Rodríguez-Arrieta, J. A. \& Sánchez-Monge, A. 2012. New records of thrips (Thysanoptera) from Central America with comments on specific characters. Florida Entomologist, 95: 1192-1193.

Ricklefs, R. E. 1984. Ecology. 9th. Edition. Chiron Press Incorporated, New York, USA. 966 pp.

Soto-Rodríguez, G. A. \& Retana-Salazar, A. P. 2003. Clave ilustrada para los géneros de Thysanoptera y especies de FranklinieIla presentes en cuatro zonas hortícolas en Alajuela, Costa Rica. Agronomía Costarricense, 27: 55-68.

Soto-Rodríguez, G. A., Retana-Salazar, A. P. \& Sanabria-Ujueta, C. 2009. Fluctuación poblacional y ecología de las especies de Thysanoptera asociadas a hortalizas en Alajuela, Costa Rica. Métodos en Ecología y Sistemática, 4: 10-28.

Valenzuela-García, R. D., Retana-Salazar, A. P., García-Martínez, O. \& Carvajal-Cazola, C. 2011. New records of thrips from Mesoamerica and comments regarding specific characters (Tubulifera: Phlaeothripidae). Florida Entomologist, 94: 372373. 